\title{
Hybrid External Fixation for Open Severe Comminuted Fractures of the Distal Femur*
}

\author{
Ebrahim Ghayem Hassankhani $^{1 \#}$, Ali Birjandinejad ${ }^{2}$, Farzad Omidi Kashani ${ }^{3}$, \\ Golnaz Ghayem Hassankhani ${ }^{4}$ \\ ${ }^{1}$ Department of Orthopedics and Spine Surgery, Orthopedic Research Center, Imam Reza Hospital, \\ Mashhad University of Medical Sciences, Mashhad, Iran \\ ${ }^{2}$ Department of Orthopedics Surgery, Shahid Kamiab Hospital, Mashhad University of Medical Sciences, Mashhad, Iran \\ ${ }^{3}$ Department of Orthopedics Surgery, Imam Reza Hospital, Mashhad University of Medical Sciences, Mashhad, Iran \\ ${ }^{4}$ Mashhad University of Medical Sciences, Mashhad, Iran \\ Email: "hasankhanie@mums.ac.ir, " eghasankhani@yahoo.com, birjandinejada@mums.ac.ir, omidif@mums.ac.ir, \\ hasankhanig@mums.ac.ir
}

Received December 17, 2012; revised January 18, 2013; accepted January 27, 2013

\begin{abstract}
Background: The treatment of distal femoral open comminuted fractures is a major problem for orthopedic surgeon. The basic and important aim in treatment of these fractures is to assemble the condylar fragments and then fix the condyles to the femoral shaft by minimum handling of the bone and soft tissues. Objectives: To evaluate the treatment of distal femoral open comminuted fractures (type C2 and C3) with hybrid external fixator. Methods: Thirty-four patients with distal femur open comminuted fracture (type C2 and C3) were treated by hybrid external fixator between January 2005 and December 2008. All of the fractures were opened with extension to joint surface. 30 patients were male and 4 were female. Their average age was 30.5 years (17 to 72 years). Average follow up period was 36 months. 12 patients had isolated fracture and 22 patients had multiple fractures. The bony and functional results were evaluated by the association for the study and application of the method of Ilizarov (ASAMI) protocol and knee society score. Results: 29 out of 34 fractures (85\%) had union without bone grafts. Average time of union was 6.1 months (4 - 19 ms). The average knee range of motion was 87.5 degrees (30 - 115 degrees). The bony results were excellent in 24 patients (70.5\%), good in 6 (17.7\%), fair in 2 (5.9\%), and poor in 2 (5.9\%), and the functional results were excellent in 10 (29.4\%) patients, good in 14 (41.2\%), fair in 6 (17.6\%), and poor in 4 (11.8\%), and the functional results were excellent in $10(29.4 \%)$ patients, good in $14(41.2 \%)$, fair in $6(17.6 \%)$, and poor in $4(11.8 \%)$ according to ASAMI protocol. According to the knee society score the functional results were excellent in 9 (26.44\%) patients, good in 13 (38.26\%), fair in 7 (20.6\%), and poor in 5 (14.7\%). Conclusion: Hybrid external fixator is an effective method for treatment of distal femoral open comminuted fractures (type C2 and C3) and may be considered as an alternative surgical methods in the management of these fractures.
\end{abstract}

Keywords: Hybrid External Fixator; Open Fractures; Distal Femoral Fracture; Type C2 and C3

\section{Background}

The treatment of distal femoral open comminuted fractures is a major problem for orthopedic surgeon. The basic and important aim in treatment of these fractures is to assemble the condylar fragments and then fix the condyles to the femoral shaft by minimum handling of the bone and soft tissues.

To reduce soft tissue trauma during surgery and fix these fractures, different surgical techniques have been developed.

These include intramedullary nailing (antegrade and

${ }^{*}$ Competing interests: The authors have no competing interests for this study.

\#Corresponding author. retrograde), plate fixation [minimally invasive percutaneous plate osteosynthesis (MIPPO), Less Invasive Stabilization System (LISS), Limited Contact Dynamic Compression Plate (LCDCP)], external Fixation (universal and ring external fixator), and hybrid external fixation [1-4].

In these types of distal femur fractures, the extent of bone and soft-tissue loss, high risk of infection, and further damage to the soft tissues precludes open reduction and internal fixation as a safe treatment method [5-7].

The hybrid external fixation preserves the soft-tissue envelope with minimal damage and allows fracture stabilization, early loading, and mobilization which promote bone healing [8]. Therefore it is an effective, safe and minimally invasive treatment for the stabilization of open 
severe comminuted fractures of the distal femur.

Purpose of this study is to determine the effectiveness and safety of hybrid external fixator in the treatment of open severe comminuted supracondylar and intercondylar fractures of distal femur.

\section{Methods}

Thirty-four patients with distal femur open comminuted fracture (type C2 and C3) were treated by hybrid external fixator between January 2005 and December 2008. This study was the result of number T-1977 research project was approved by MUMS ethic committee. Average follow up was 36 months. Thirty patients were male and 4 were female. Their average age was 30.5 years (17 to 72 years). According to the OTA classification, 20 fractures were type C2 and 14 were type C3. All fractures were open and extended to joint surface. The open fractures were classified as type IIIA (27 cases), and type III B (7 cases) according to the systems of Gustilo and Anderson (Table 1).

Twelve patients had an isolated fracture and 22 patients had multiple fractures.

Four patients were of the type III C fractures and underwent above knee amputation because of severe crush, and were excluded from the study.

Our protocol in all patients was Wound debridement after admission to the emergency department. In debridement protocol, $10 \mathrm{~L}$ of saline solution was used for irrigation. In debridement excision of all necrotic and suspect bone fragments were done. After debridement, the hybrid ring external fixator was applied. Plastic surgeons were included in the treatment team when needed. Early coverage of the exposed bone was achieved by local flaps or free-tissue transfer within 15 days of injury. The hybrid external fixator frame was assembled preoperatively for all patients.

All operations were carried out on a traction table with the patient supine. During the operation, continuous traction was applied with a pin inserted in the tibia with the knee in $45^{\circ}$ to $60^{\circ}$ flexion.

A gentle manipulation was applied and alignment was checked by fluoroscopy. If the position was acceptable, the frame was applied in a closed manner; if not, we used minimal open reduction and internal fixation with screws (cannulated) or Kirschner wires from the wound or by extending it.

Two or three olive pins were placed in the distal femur from the anterolateral to posteromedial and from an teromedial to posterolateral directions and each pin were fixed to an appropriate ring.

When the ring was constructed, the wires were tensioned and tightened, then three half pins were inserted in different planes in the proximal femur, and connected
Table 1. Clinical data of 34 patients with open comminuted distal femur fracture treated by hybrid external fixator.

\begin{tabular}{|c|c|}
\hline Data & Number of patients \\
\hline \multicolumn{2}{|c|}{ Type of fracture (Gustilo classification) } \\
\hline Type III A & 27 \\
\hline Type III B & 7 \\
\hline \multicolumn{2}{|c|}{ Type of fracture (OTA classification) } \\
\hline Type C2 & 20 \\
\hline Type C3 & 14 \\
\hline \multicolumn{2}{|c|}{ Sex } \\
\hline Female & 4 \\
\hline Male & 30 \\
\hline \multicolumn{2}{|c|}{ Other treatments } \\
\hline Bone graft & 2 \\
\hline Bone graft + Bon transport & 3 \\
\hline \multicolumn{2}{|c|}{ Complications } \\
\hline Infection & 11(Superficial)-7(Profound) \\
\hline Residual deformity & 5 \\
\hline Shortening more than $2 / 5 \mathrm{~cm}$ & 0 \\
\hline Returned to previous work & 20 \\
\hline Changed work & 5 \\
\hline Unable work & 3 \\
\hline Significant pain & 8 \\
\hline Noticeable limp & 11 \\
\hline Stiff joint & 12 \\
\hline
\end{tabular}

to the distal femur ring. The final reduction was checked and finally all connections were retightened (Figures 1 and 2).

In order to obtain a secure fixation, the frame was extended to the proximal tibia if needed. In three patients the frame was extended to the proximal tibia due to obtain secure fixation.

Additional procedures such as wire exchange (because of pin site infection), frame adjustments for alignment, debridement of necrotic or infected tissues and skin graft were included.

Patients permitted exercises and ambulation as soon as pain subsided for prevention of contractures and stiffness 


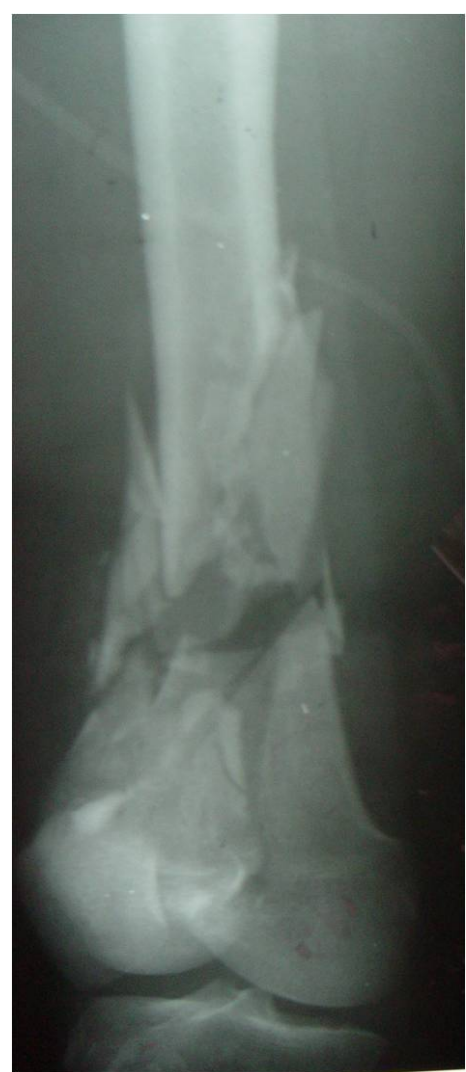

(a)

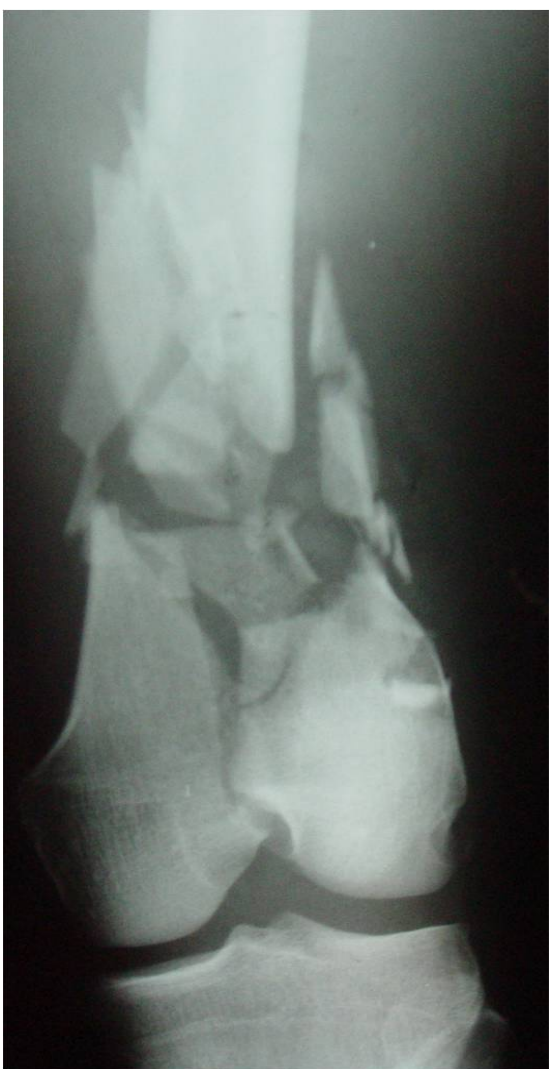

(b)

Figure 1. A 36 years old man with open (type IIIA of Gustilo classification) severe comminuted fractures of right distal femur (type C2 of OTA classification) due to motor cycle accident.

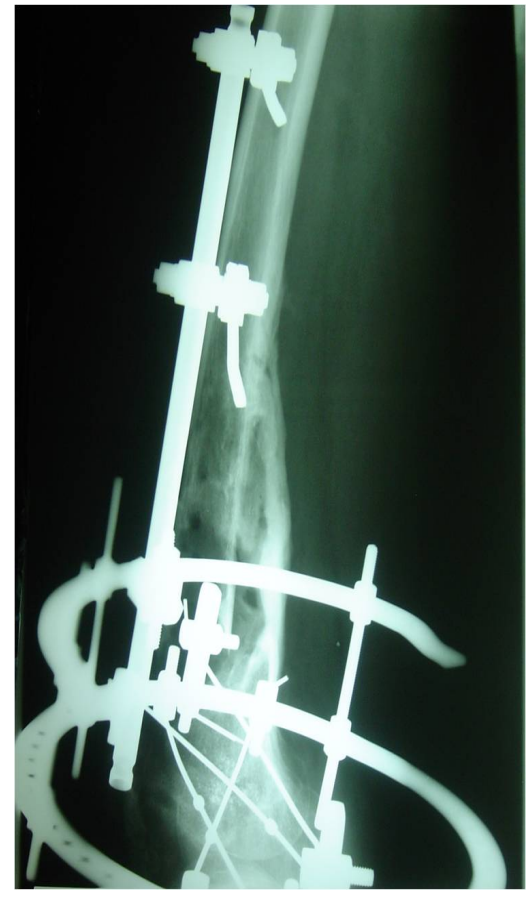

(a)

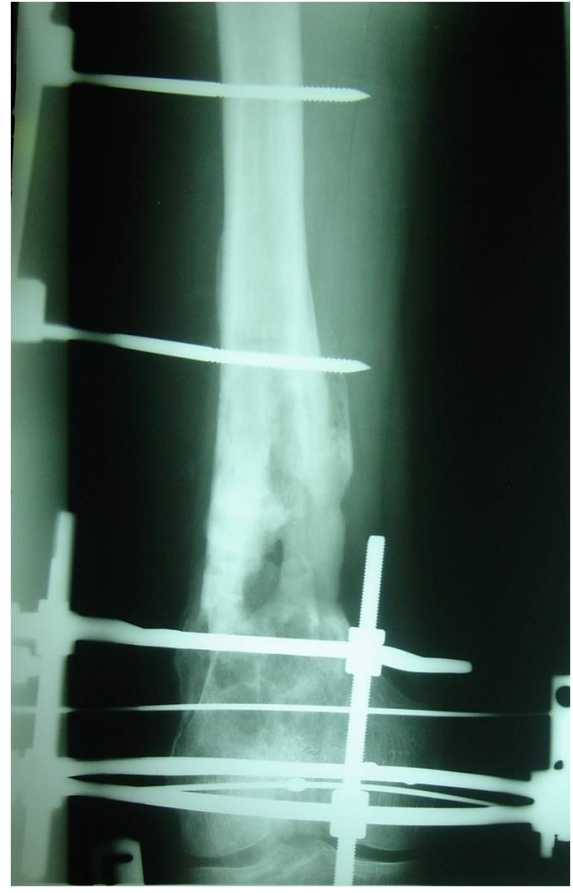

(b)

Figure 2. After 4months of debridement, reduction, minimally internal fixation (with screw) and application of hybrid external fixation (no other operation). 
of joints. Partial weight-bearing continued after 6 - 8 weeks until there was satisfactory evidence of callus formation.

We followed up patients regularly at intervals of 2 weeks until the frame was removed and then monthly. At each follow-up, the reduction and healing of the fracture was assessed radiologically. The mean time of hybrid external fixator removal was 30 weeks (16 - 62 weeks). We usually removed the apparatus later than for more consolidation of bony union (Figure 3).

The bony and functional results were evaluated by ASAMI score and functional results by knee society score at the end of follow-up (Table 2).

\section{Results: (Table 1)}

According to the association for the study and application of the method of Ilizarov (ASAMI) protocol, the results were evaluated as bony and functional results.

The criteria for determination of bony results were: union, infection, residual deformity and limb length discrepancy and for functional results were: limping, stiffness of either the knee or the ankle, sympathetic dystrophy, pain that resulted in reduced activity or disturbed sleep and inactivity (inability to return to previous activities).

Functional results also were evaluated by knee society score.

Union was achieved in 29 patients without bone grafting. Bone grafting was necessary in 2 cases, and bone transport with bone grafting was done in 3 patients with $2 \mathrm{~cm}$ defect.

All fractures united at a mean of 24.4 weeks (16 - 76 weeks). 27 patients had union within 24 weeks or less.

Five patients united with some degrees of malalignment including: 2 patients with 8 degrees of valgus, 2 patients with 11 degrees external rotation, and 1 patient with 9 degrees recurvatum .two patients had $1.5-2.5 \mathrm{~cm}$ shortening.

Pin-track infections occurred in 11 patients and resolved after oral antibiotics and care of the pin sites.

No patient required a secondary procedure because of loosening of the frame with loss of reduction. Seven (20\%) patients had fracture site infection effectively controlled by drainage, debridement, antibiotic therapy and antiseptic dressings. Two patients had skin graft with no need to plastic surgeon.

The limitation of knee range of motion occurred in all

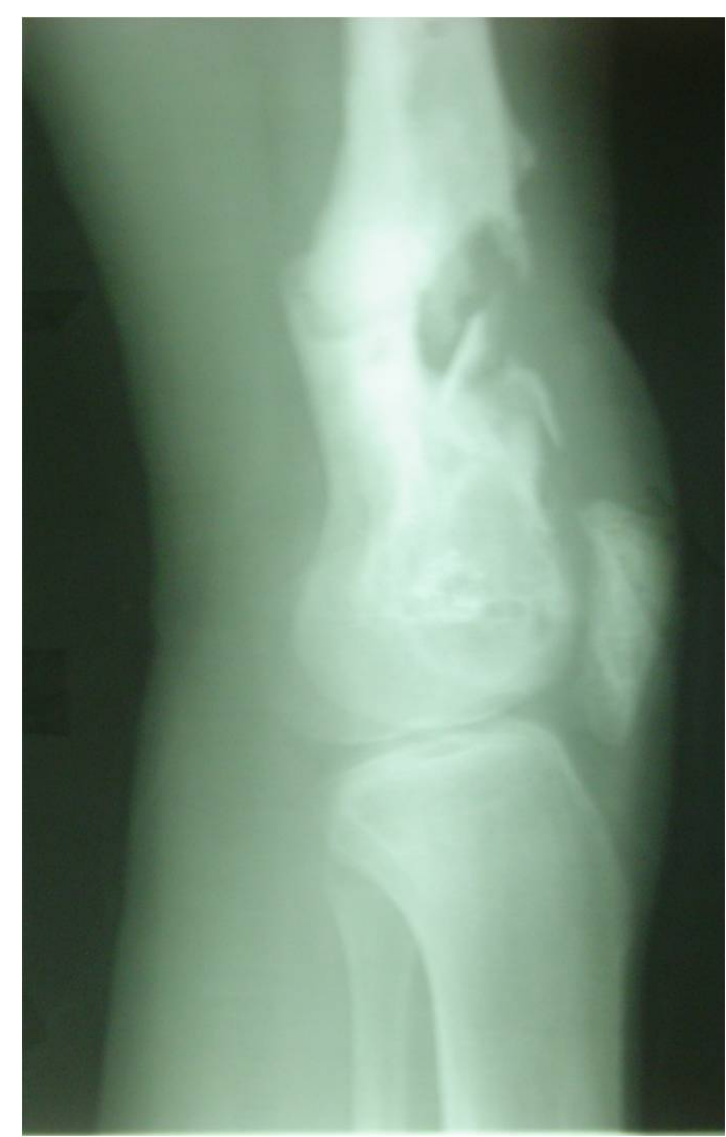

(a)

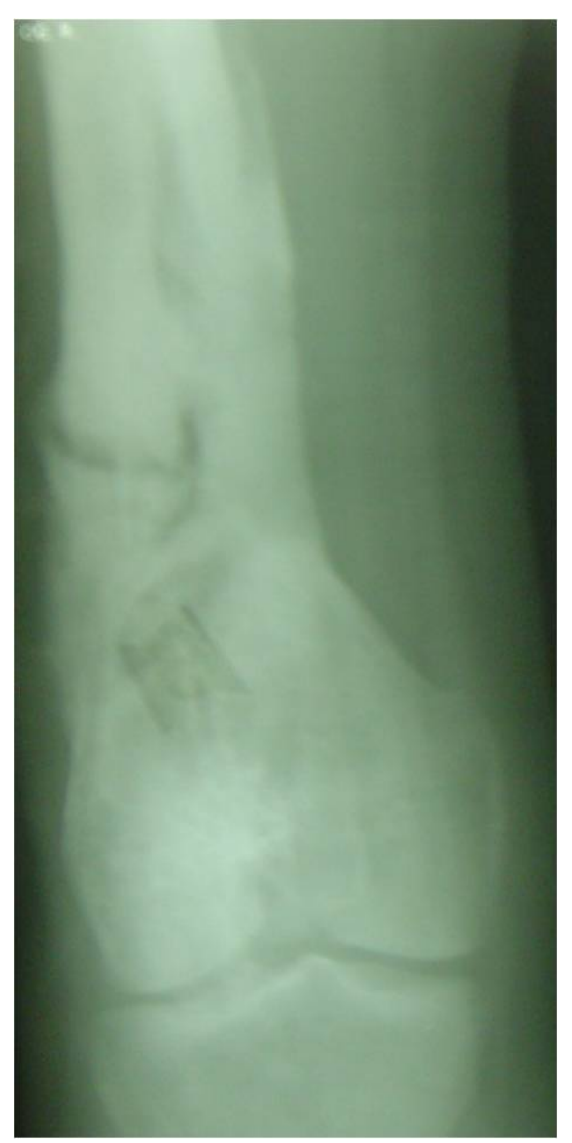

(b)

Figure 3. After 8 months and removal of hybrid external fixation apparatus with good bony union. 
Table 2. Classification of the results according to the modified ASAMI protocol.

\begin{tabular}{|c|c|c|}
\hline & Bone result & Functional result \\
\hline \multirow{3}{*}{ Excellent } & Bone union, no infection deformity $<7^{\circ}$ & $\begin{array}{l}\text { Ability to perform previous activities of daily living (ADL), no } \\
\text { pain or mild pain }\end{array}$ \\
\hline & \multirow{2}{*}{ LLD (lower limb discrepancy) $<2.5 \mathrm{~cm}$} & $\begin{array}{l}\text { No limp, no soft tissue sympathetic dystrophy knee or ankle joint } \\
\text { contracture }<5^{\circ}\end{array}$ \\
\hline & & Loss of ankle or knee motion $<15^{\circ}$ \\
\hline \multirow{3}{*}{ Good } & \multirow{3}{*}{ Bone union, failure to meet one of the other criteria } & Almost all ADL with minimal difficulty \\
\hline & & \\
\hline & & No pain or mild pain, failure to meet on of the other criteria \\
\hline \multirow{2}{*}{ Fair } & \multirow{2}{*}{ Bone union, failure to meet two of the other criteria } & Most ADL with minimal difficulty \\
\hline & & No pain or mild pain, failure to meet two of the other criteria \\
\hline \multirow[b]{2}{*}{ Poor } & \multirow[b]{2}{*}{$\begin{array}{l}\text { Nonunion or refracture, failure to meet three of the } \\
\text { other criteria }\end{array}$} & Significantly limited ADL \\
\hline & & $\begin{array}{l}\text { Significantly pain requiring narcotics, failure to meet three of the } \\
\text { other criteria }\end{array}$ \\
\hline
\end{tabular}

34 patients. The final average knee range of motion was 87.5 degrees (30 - 115 degrees). The limited range of motion was in flexion and the extension was near normal in all patients. The limitation of knee range of motion was greatest in patients with a type C3 fracture. Eight patients required quadriceps plasty to increase the range of knee motion. At final follow-up, 8 patients had $<90$ degrees of knee flexion, 12 patients $=90$ degrees, and 14 patients $>90$ degrees. Eight patients had significant pain requiring analgesics.

Twelve patients had stiffness of the knee. Eleven patients had noticeable limping. Fourteen patients achieved ability to perform previous activities of daily living (ADL) and returned to their previous work, 12 patients got ability to perform previous activities of daily living (ADL) and previous work with minimal difficulty and 8 patients had significantly limited activities of daily living (ADL), 5 patients required a change from previous work and 3 patients have not returned to any work till now.

The bony results were excellent in 24 patients (70.5\%), good in 6 (17.7\%), fair in 2 (5\%/9\%), and poor in 2 (5.9\%), and functional results were excellent in 10 (29.4\%) patients, good in $14(41.2 \%)$, fair in $6(17.6 \%)$, and poor in $4(11.8 \%)$ according to ASAMI protocol (Table 3).

According to the knee society score the functional results were excellent in $9(26.44 \%)$ patients, good in 13 (38.26\%), fair in 7 (20.6\%), and poor in 5 (14.7\%).

\section{Discussion}

The treatment of distal femoral open comminuted fractures (type C2 and C3 of OTA classification), particu- larly when associated with bone defect and soft tissue crush, is difficult and a major problem for orthopedic surgeons. In the present study, we found that the hybrid external fixator is an excellent method for open type C2 and C3 fractures of the distal femur especially with comminution.

In these types of fractures, the advantages of the hybrid external fixator are, principally, the minimal surgical trauma and bleeding, early weight-bearing, low incidence of deep infections, and possibility to perform progressive corrections and soft tissue care during the treatment course $[9,10]$.

The hospitalization time is decreased with this method with concomitant decrease in hospital costs.

Although there are various methods of treatment for comminuted fractures of the distal femur (type C2 and C3 of OTA classification) including intramedullary nailing (antegrade and retrograde), plate fixation [minimally invasive percutaneous plate osteosynthesis (MIPPO), less Invasive stabilization system (LISS), limited contact dynamic compression plate (LC-DCP)], external fixation (universal and ring external fixator), and hybrid external fixation [1-4], but when these fracture are associated with open soft tissues injury, the hybrid external fixator could be the treatment of choice.

The indications for using hybrid external fixator in distal femoral fractures may be considered as followings: comminuted fractures in which stable reduction cannot be obtained by open reduction and internal fixation, those with open soft tissues injury and bone loss, fractures in osteoporotic bone, fractures in multiply injured patients, and combination of above mentioned conditions [11]. 
Table 3. The bony and functional results of 34 patients with open comminuted distal femur fracture treated by hybrid external fixator.

\begin{tabular}{ccccc}
\hline \multirow{2}{*}{ Grade } & \multicolumn{2}{c}{ Bone results } & \multicolumn{2}{c}{ Functional results } \\
\cline { 2 - 5 } & Number of patients & Percent & Number of patients & Percent \\
\hline Excellent & 24 & 70.5 & 10 & 29.4 \\
Good & 6 & 17.7 & 14 & 41.2 \\
Fair & 2 & 5.9 & 6 & 17.7 \\
Poor & 2 & 5.9 & 34 & 11.7 \\
Total & 34 & 100 & 34 & 100 \\
\hline
\end{tabular}

Recently, many orthopedic surgeons have reported alternative methods of stabilization of comminuted distal femur fractures including intramedullary nailing and plate fixation with good results. In these studies, all of the reported cases were closed comminuted fractures of the distal femur with low risk, contrary to our open high-risk case series [1,12-14].

This study showed that with the hybrid external fixator, open comminuted distal femoral fractures (type C2 and C3 of OTA classification) can be managed successfully because of minimal or no surgical exposure ,shorter operation time, minimum blood loss, minimal handling of bone and soft tissue with fast healing of the fracture and greater stability [15-18].

None of the above mentioned techniques (intramedullary nailing and plate fixation) affords the surgeon capabilities of correcting deformities, regenerating new bone without the use of bone graft, progressive lengthening the extremity, permit bone transport for bone defect filling and allow weight bearing very soon.

The present study and many other studies confirm that all of these capabilities are possible with application of the hybrid external fixator.

This study also showed that the hybrid external fixator has the ability of successful managing of the fracture, soft-tissue defect, infection, and bone loss simultaneously $[19,20]$.

Although the classical Ilizarov method (ring external fixator) is an acceptable method for healing of docking site by continuous and gradual compression, as many other surgeons, we found that autogenous bone graft accelerates healing $[19,21,22]$.

In our study, 5 patients had autogenous bone graft at the docking site and in all patients bone union was obtained so we recommend bone graft at the docking site in order to accelerate union, reduce external fixation time and to avoid refracture.

We treated most fractures in this series by ligamentotaxis and closed reduction, as in

Ilizarov's original description [23] but in cases with failure to obtain anatomical reduction by ligament taxis, limited open reduction and minimal internal fixation was performed before the application of the frame in severely comminuted fractures which extend into the joint [1124].

Nonunion of supracondylar fractures of the distal femur is unusual, but may follow open reduction and internal fixation. In a retrospective review of ununited supracondylar fractures, all of them had been treated by open reduction and internal fixation [25]. We had only 2 patients with nonunion and in no other patients bone grafting was required. Similar low incidences of nonunion have been reported in other series using external fixator $[9,11,21,26]$.

The limitation of knee motion was the most disabling complication in our study, as also reported in other studies $[9,11,27,28]$. There are several factors that cause limitation of knee motion including: type of the fracture (type C3 of OTA classification), severity of the associated soft-tissue injury (type IIIB and type IIIC of Gustilo classification) and incorrect application of external fixator (the knee in extension). We carried out the application of distal wires in a position of moderate knee flexion, in accordance with Ilizarov's original technique, thus stretching the extensor mechanism of the knee [16].

Although, our cases were complicated in comparison with other studies that have been used the Ilizarov, intramedullary nailing, or plate system for fixation, the results were the same or even better in our studies [5-7, $12,15,24]$.

\section{Conclusion}

Hybrid external fixator is an effective method for treatment of distal femoral open comminuted fractures (type C2 and C3 of OTA classification) particularly when associated with bone defect and soft tissue crush and may be considered as an alternative surgical methods in the management of these complex fractures.

\section{Authors' Contributions}

Ebrahim ghayem Hassankhani: participated in the sequence alignment and drafted the manuscript, Performed the statistical analysis. 
Ali birjandi nejad: participated in the design of the study.

Farzad Omidi kashani: participated in the design of the study.

Golnaz ghayem Hassankhani: participated in its design and coordination.

\section{Acknowledgements}

The authors thank orthopaedic research center group and Dr. M.Ebrahim zadeh for their assistance.

\section{REFERENCES}

[1] F. Walcher, J. Frank and I. Marzi, "Retrograde Nailing of Distal Femoral Fracture Clear and Potential Indications," European Journal of Trauma, Vol. 26, No. 4, 2000, pp. 155-168.

[2] C. Krettek, P. Schandelmaier, T. Miclau, R. Bertram, W. Holmes and H. Tscherne, "Transarticular Joint Reconstruction and Indirect Plate Osteosynthesis for Complex Distal Supracondylar Femoral Fractures,” Injury, Vol. 28, No. S1, 1997, pp. A31-A41. doi:10.1016/S0020-1383(97)00076-4

[3] A. Kumar, V. Jasani and M. Butt, "Management of Distal Femoral Fractures in Elderly Patients Using Retrograde Titanium Supracondylar Nails,” Injury, Vol. 31, No. 3, 2000, pp. 169-173. doi:10.1016/S0020-1383(99)00275-2

[4] K. Leung, W. Shen, L. Mui and A. Grosse, "Interlocking Intramedullary Nailing for Supracondylar and Intercondylar Fractures of the Distal Part of the Femur," The Journal of Bone and Joint Surgery, Vol. 73, No. 3, 1991, pp. 332-340.

[5] A. Lerner and H. Stein, "Hybrid Thin Wire External Fixation: An Effective, Minimally Invasive, Modular Surgical Tool for the Stabilization of Periarticular Fractures," Orthopedics, 2004, Vol. 27, No. 1, pp. 59-62.

[6] P. J. Kregor, "Distal Femur Fractures with Complex Articular Involvement: Management By Articular Exposure and Submuscular Fixation," Orthopedic Clinics of North America, Vol. 33, No. 1, 2002, pp. 153-175. doi:10.1016/S0030-5898(03)00078-6

[7] A. Haluk, R. Ali, Z. Gurhan and E. Gurkan, "Treatment of Intraarticular Comminuted Supracondylar Femoral Fractures by Indirect Plate Osteosynthesis," Acta Orthopaedica et Traumatologica Turcica Journal, Vol. 36, No. 5 2002.

[8] M. Arazi, R. Memik, T. C. Ögün and M. Yel, "Ilizarov External Fixation for Severely Comminuted Supracondylar and Intercondylar Fractures of the Distal Femur," Journal of Bone and Joint Surgery, Vol. 83B, No. 5, 2001, pp. 663-667. doi:10.1302/0301-620X.83B5.11520

[9] J. L. Marsh, H. Jansen, H. K. Yoong and E. M. Found Jr., "Supracondylar Fractures of the Femur Treated by External Fixation,” Journal of Orthopaedic Trauma, Vol. 11, No. 6, 1997, pp. 405-411. doi:10.1097/00005131-199708000-00004

[10] F. Ali and M. Saleh, "Treatment of Isolated Complex Dis- tal Femoral Fractures by External Fixation,” Injury, Vol. 31, No. 3, 2000, pp. 139-146. doi:10.1016/S0020-1383(99)00249-1

[11] J. J. Hutson Jr. and G. A. Zych, "Treatment of Comminuted Intraarticular Distal Femur Fractures with Limited Internal and External Tensioned Wire Fixation,” Journal of Orthopaedic Trauma, Vol. 14, No. 6, 2000, pp. 405413. doi:10.1097/00005131-200008000-00005

[12] F. C. Kao, Y. K. Tu, J. Y. Su, K. Y. Hsu, C. H. Wu and M. C. Chou, "Treatment of Distal Femoral Fracture by Minimally Invasive Percutaneous Plate Osteosynthesis: Comparison between the Dynamic Condylar Screw and the Less Invasive Stabilization System,” Journal of Trauma and Acute Surgery, Vol. 67, No. 4, 2009, pp. 719-726. doi:10.1097/TA.0b013e31819d9cb2

[13] W. Kolb, H. Guhlmann, C. Windisch, F. Marx, K. Kolb and H. Koller, "Fixation of Distal Femoral Fractures with the Less Invasive Stabilization System: A Minimally Invasive Treatment with Locked Fixed-Angle Screws," Journal of Trauma and Acute Surgery, Vol. 65, No. 6, 2008, pp. 1425-1434. doi:10.1097/TA.0b013e318166d24a

[14] I. H. Jeon, C. W. Oh, S. J. Kim, B. C. Park, H. S. Kyung and J. C. Ihn, "Minimally Invasive Percutaneous Plating of Distal Femoral Fractures Using the Dynamic Condylar Screw,” Journal of Trauma and Acute Surgery, Vol. 57, No. 5, 2004, pp. 1048-1052. doi:10.1097/01.TA.0000100373.54984.75

[15] J. J. Hutson Jr., "Reconstruction of Distal Intercondylar Femoral Fractures with Limited Internal Fixation and Ilizarov Tensioned-Wire External Fixation,” Techniques in Orthopaedics, Vol. 11, No. 2, 1996, pp. 182-195. doi:10.1097/00013611-199601120-00010

[16] G. A. Ilizarov, "The Transosseous Osteosynthesis: Theoretical and Clinical Aspects of the Regeneration and Growth of Tissue,” Springer-Verlag, Berlin, 1992.

[17] M. Kocaoglu, "Ilizarov Applications in Skeletal Trauma," In: M. Cakmak and M. Kocao glu, Eds., Surgical Principles of the Ilizarov Technique Istanbul, Doruk Grafik, Istanbul, 1999, pp. 183-208.

[18] S. A. Green, “The Ilizarov Method,” In: B. D. Browner, J. B. Jupiter, A. M. Levine and P. G. Trafton, Eds., Skeletal Trauma: Fractures, Dislocations, Ligamentous Injuries, WB Saunders, Philadelphia, 1997, pp. 661-701.

[19] E. Ghayem Hassankhani, T. Payvandi Mahamad and A. Birjandinejad, "The Ilizarov Ring External Fixator in Complex Open Fractures of the Tibia,” European Journal of Trauma, Vol. 31, 2006, pp. 1-7.

[20] A. Saridis, E. Panagiotopoulos, M. Tyllianakis, C. Matzaroglou, N. Vandoros and E. Lambiris, "The Use of the Ilizarov Method as a Salvage Procedure in Infected NonUnion of the Distal Femur with Bone Loss," Journal of Bone and Joint Surgery, Vol. 88B, No. 2, 2006, pp. 232237.

[21] M. Lalit, C. Manish and V. Jashan, "The Ilizarov Method in Infected Non-Union of Fractures," Journal of Injury, Vol. 31, No. 7, 2000, pp. 509-517.

[22] D. R. Marsh, S. Shah and J. Elliot, "The Ilizarov Method in Non-Union, Mal-Union and Infection of Fractures," 
Journal of Bone and Joint Surgery, Vol. 79B, 1997, pp. 273-279. doi:10.1302/0301-620X.79B2.6636

[23] G. A. Ilizarov, "The Transosseous Osteosynthesis: Theoretical and Clinical Aspects of the Regeneration and Growth of Tissue,” Springer-Verlag, Berlin, 1992.

[24] J. J. Hutson Jr., "Reconstruction of Distal Intercondylar Femoral Fractures with Limited Internal Fixation and Ilizarov Tensioned-Wire External Fixation,” Techniques in Orthopaedics, Vol. 11, No. 2, 1996, pp. 182-195. doi:10.1097/00013611-199601120-00010

[25] M. W. Chapman and C. G. Finkemeier, "Treatment of Supracondylar Non-Unions of the Femur with Plate Fixation and Bone Graft,” Journal of Bone and Joint Surgery, Vol. 81, No. 9, 1999, pp. 1217-1228.
[26] K. Aktuglu, H. Önçag and S. Kara, "Ilizarov Treatment of Supracondylar and Intercondylar Fractures of the Distal Femur,” In: M. Çakmak and M. Kocaoglu, Eds., External Fixation, Damla Matbaasi, Istanbul, 1995, pp. 175-182.

[27] M. Kocaoglu, "Ilizarov Applications in Skeletal Trauma," In: M. Cakmak and M. Kocaoglu, Eds., Surgical Principles of the Ilizarov Technique, Doruk Grafik, Istanbul, 1999, pp. 183-208.

[28] A. Kutlu, M. Yel, M. Mutlu, M. I. S. Kapicioglu, "Fractures of the Femoral Shaft with Management of External Fixation to Experience Some Problems,” In: M. Cakmak and M. Kocaoglu, Eds., External Fixation, Damla Matbaasi, Istanbul, 1995, pp. 166-169. 